\title{
Biomarkers of infection for the differential diagnosis of pleural effusions
}

\author{
J.M. Porcel*, M. Vives”, G. Caoॠ, S. Bielsa*, A. Ruiz-González*, \\ A. Martínez-Iribarren" and A. Esquerda"
}

ABSTRACT: We aimed to investigate whether pleural fluid concentrations of biomarkers for bacterial infection, namely triggering receptor expressed on myeloid cells (sTREM-1), procalcitonin (PCT), lipopolysaccharide-binding protein (LBP) and C-reactive protein (CRP), might identify infectious effusions and discriminate between complicated (CPPEs) and uncomplicated parapneumonic effusions (UPPEs).

Stored pleural fluid samples from 308 patients with different causes of pleural effusion were used to measure the four biomarkers. Receiver-operating characteristic analysis determined the accuracy of the new tests.

Median pleural fluid levels of CRP, STREM-1 and LBP were significantly higher in CPPE compared with those in other aetiologies. The area under the curve for distinguishing infectious (parapneumonics and tuberculosis) from noninfectious effusions was 0.87 for CRP, 0.86 for sTREM-1, 0.57 for PCT and 0.87 for LBP. Regarding the discrimination of nonpurulent CPPE versus UPPE, a multivariate analysis found that pleural fluid glucose $\leqslant 60 \mathrm{mg} \cdot \mathrm{dL}^{-1}$, LBP $\geqslant 17 \mu \mathrm{g} \cdot \mathrm{mL}^{-1}$ and CRP $\geqslant 80 \mathrm{mg} \cdot \mathrm{L}^{-1}$ were the best parameters. Individually, none of the new biomarkers achieved better performance characteristics than $\mathrm{pH}$, glucose or lactate dehydrogenase in labelling CPPE.

In conclusion, elevated pleural fluid levels of CRP, STREM and LBP identify patients with infectious effusions, particularly those with CPPE. PCT has no value for the differential diagnosis of pleural effusions.

KEYWORDS: C-reactive protein, empyema, lipopolysaccharide-binding protein, pleural effusion, procalcitonin, triggering receptor expressed on myeloid cells

$\mathbf{R}$ apid and accurate diagnosis remains the major clinical challenge in patients with suspected pleural infection. Since clinical data may be elusive, measurement of biomarkers in pleural fluids might provide a reliable tool for estimating the probability of an infection [1]. In addition, the search for biomarkers of poor prognosis in parapneumonic effusions (PPEs) is critical for better identifying patients in which drainage of the pleural space is mandatory [2].

In general, $\mathrm{C}$-reactive protein (CRP) is considered to be the biomarker of choice to detect an inflammatory state, whether or not it is triggered by an infection. Newer circulating markers, such as soluble triggering receptor expressed on myeloid cells (sTREM-1), procalcitonin (PCT) and lipopolysaccharide-binding protein (LBP), seem to have a pivotal role in the inflammatory host response to microbial infections [3, 4]. Therefore, we hypothesised that when measured in pleural fluid, they might be useful for diagnosing or ruling out infectious effusions and for identifying complicated parapneumonic effusions (CPPEs)

The aims of the present study were to measure the pleural fluid concentrations of CRP, sTREM, PCT and LBP in patients with different causes of pleural effusions and to explore their usefulness in both predicting an infectious aetiology and discriminating between subgroups of PPEs.

\section{METHODS}

\section{Study population}

Since 1994, we have been prospectively maintaining a database on all patients who undergo diagnostic thoracentesis at the Arnau de Vilanova University Hospital (Lleida, Spain), as well as a pleural bank of fluid specimens that are processed and stored at $-80^{\circ} \mathrm{C}$. The indication for diagnostic thoracentesis was the presence of a clinically significant pleural effusion of uncertain origin. In patients submitted to more than one
AFFILIATIONS

*Pleural Disease Unit, Dept of Internal Medicine

"Dept of Laboratory Medicine, Arnau de Vilanova University Hospital, IRBLLEIDA, Lleida, and

${ }^{\text {\#}}$ Service of Internal Medicine, Clinica Recoletas, Albacete, Spain.

CORRESPONDENCE

J.M. Porcel

Dept of Internal Medicine Arnau de Vilanova University Hospital Avda Alcalde Rovira Roure 80 25198 Lleida

Spain

E-mail: jporcelp@yahoo.es

Received:

Dec 292008

Accepted after revision:

June 052009

First published online:

June 182009 
thoracentesis during the hospitalisation period, only the results of the first tap were considered. Samples of pleural fluid were immediately analysed for routine biochemistries (e.g. $\mathrm{pH}$, glucose and lactate dehydrogenase (LDH)), while supernatants were frozen within $4 \mathrm{~h}$ of collection until the measurement of the biomarkers at the end of the study.

For the present study, 308 pleural fluid samples collected from 2004 to 2008 were randomly selected for biomarkers determination. Patients were classified into seven groups according to the cause of the pleural effusion: transudates (40 patients), malignant effusions (40 patients), tuberculous pleurisy (50 patients), uncomplicated parapneumonics (UPPE; 60 patients), nonpurulent CPPE (68 patients), empyemas (30 patients) and miscellaneous exudates (20 patients). Primary tumours in malignant effusions were lung (14 patients), breast (eight patients), unknown primary (six patients), lymphoma (four patients), ovary (three patients), gastrointestinal (three patients) and mesothelioma (two patients). Among the miscellaneous exudates, there were five post-traumatic, three idiopathic, three post-coronary artery bypass surgery, two pericarditis, two pulmonary embolism, two abdominal abscess, one pancreatitis, one post-abdominal surgery and one systemic lupus erythematosus.

The local Ethics Committee (Arnau de Vilanova University Hospital, Lleida, Spain) approved this study, and all patients gave informed consent for the analysis of stored specimens for future research.

\section{Diagnostic criteria}

A pleural effusion was categorised as malignant if malignant cells were detected on cytological examination of the pleural fluid or biopsy specimen. Tuberculous pleuritis was diagnosed if Löwenstein cultures of pleural fluid, sputum or pleural biopsy tissue samples were positive (26 patients), a pleural biopsy specimen showed granulomas in the parietal pleura (five patients) or an exudative lymphocytic effusion with high adenosine deaminase levels $\left(>40 \mathrm{U} \cdot \mathrm{L}^{-1}\right)$ cleared in response to antituberculous therapy (19 patients). Transudates were effusions secondary to heart failure (30 patients), cirrhosis (six patients), nephrotic syndrome (two patients) or atelectasis (two patients). PPEs referred to those associated with bacterial pneumonia and were classified into three groups: UPPE (resolution with antibiotic treatment alone), nonpurulent CPPE (requirement of an invasive procedure such as tube thoracostomy for cure) and empyema (pus into the pleural space). Other causes of pleural effusions were determined by well-established clinical criteria.

\section{Measurement of biomarkers}

Assays for the four inflammatory markers, namely CRP, STREM, PCT and LBP, were performed on the stored cell-free supernatants of pleural fluid samples. All samples were tested in a random order and by technicians who were blinded to the clinical diagnosis of patients at the end of the study.

Pleural fluid CRP was measured using a particle-enhanced immunoturbidimetric assay (CRPLX, Tina-quant; Roche Diagnostics $\mathrm{GmbH}$, Mannheim, Germany) on Roche automated clinical chemistry analysers. The sTREM-1 concentrations in pleural fluid were measured by a sandwich ELISA (DuoSet ELISA; R\&D Systems Europe, Abingdon, UK), PCT by a two-site immunoluminometric assay (Liaison Brahms PCT; DiaSorin, Saluggia, Italy) and LBP with a solid-phase, enzymelabelled chemiluminescent immunometric assay (Immulite 2000 LBP; Siemens, Los Angeles, CA, USA), according to the manufacturer's instructions. The samples for sTREM-1 were assayed in duplicate.

\section{Data analysis}

Continuous data are presented as median (quartile range). Between-group comparisons were performed with KruskalWallis tests and Dunn's multiple comparison post hoc tests for

TABLE 1 Demographics and pleural fluid biomarkers in patients

\begin{tabular}{|c|c|c|c|c|c|c|c|c|}
\hline & Transudates & Malignant & Tuberculosis & UPPE & CPPE & Empyema & $\begin{array}{l}\text { Miscellaneous } \\
\text { exudates }\end{array}$ & p-value ${ }^{\#}$ \\
\hline Subjects n & 40 & 40 & 50 & 60 & 68 & 30 & 20 & \\
\hline Age yrs & $78(67-83)$ & $72(62-79)$ & $38(27-52)^{\bullet}$ & $66(43-78)$ & $50(40-64)^{\bullet}$ & $53(41-72)^{\bullet}$ & $70(43-79)$ & $<0.001$ \\
\hline $\mathrm{CRP} \mathrm{mg} \cdot \mathrm{L}^{-1}$ & $3(1-9)^{n}$ & $8(4-20)^{\bullet}$ & $37(19-59)$ & 41 (21-82) & $105(74-150)^{+}$ & $91(24-141)^{+}$ & $25(9-47)$ & $<0.001$ \\
\hline $\begin{array}{l}\text { sTREM-1 } \\
\mathrm{pg} \cdot \mathrm{mL}^{-1}\end{array}$ & $4(0-23)^{\circ}$ & $18(4-58)^{\circ}$ & $96(61-181)$ & $77(36-158)$ & $279(145-444)^{+}$ & $515(251-785)^{+}$ & $39(0-95)^{\bullet}$ & $<0.001$ \\
\hline PCT $\mathrm{ng} \cdot \mathrm{mL}^{-1}$ & $0.15(0.08-0.24)$ & $0.10(0.05-0.25)$ & $0.10(0.07-0.26)$ & $0.19(0.07-0.51)$ & $0.26(0.14-0.63)$ & $0.19(0.06-0.63)$ & $0.33(0.11-0.50)$ & $<0.001$ \\
\hline $\mathrm{LBP} \mu \mathrm{g} \cdot \mathrm{mL}^{-1}$ & $1.8(0-3.4)^{\circ}$ & $4.8(2.8-6.8)^{\bullet}$ & $12.5(8.5-15.2)$ & $10.3(6.6-15.6)$ & $25(17.3-38.9)^{+}$ & $17.7(7.5-29)$ & $7(4.4-11.4)$ & $<0.001$ \\
\hline
\end{tabular}

Data are presented as median (quartile range) or $n(\%)$, unless otherwise indicated. UPPE: uncomplicated parapneumonic effusion; CPPE: complicated parapneumonic effusion; CRP: C-reactive protein; sTREM-1: soluble triggering receptor expressed on myeloid cells; PCT: procalcitonin; LPB: lipopolysaccharide-binding protein. * : Chisquared with post hoc analysis of adjusted residuals (qualitative variables) or Kruskal-Wallis test with post hoc Dunn test (quantitative variables); ": significantly lower than the respective values in other groups by post-hoc test $(p<0.05) ;^{+}$: significantly higher than the respective values in other groups by post hoc test $(p<0.05)$. $n=308$. 

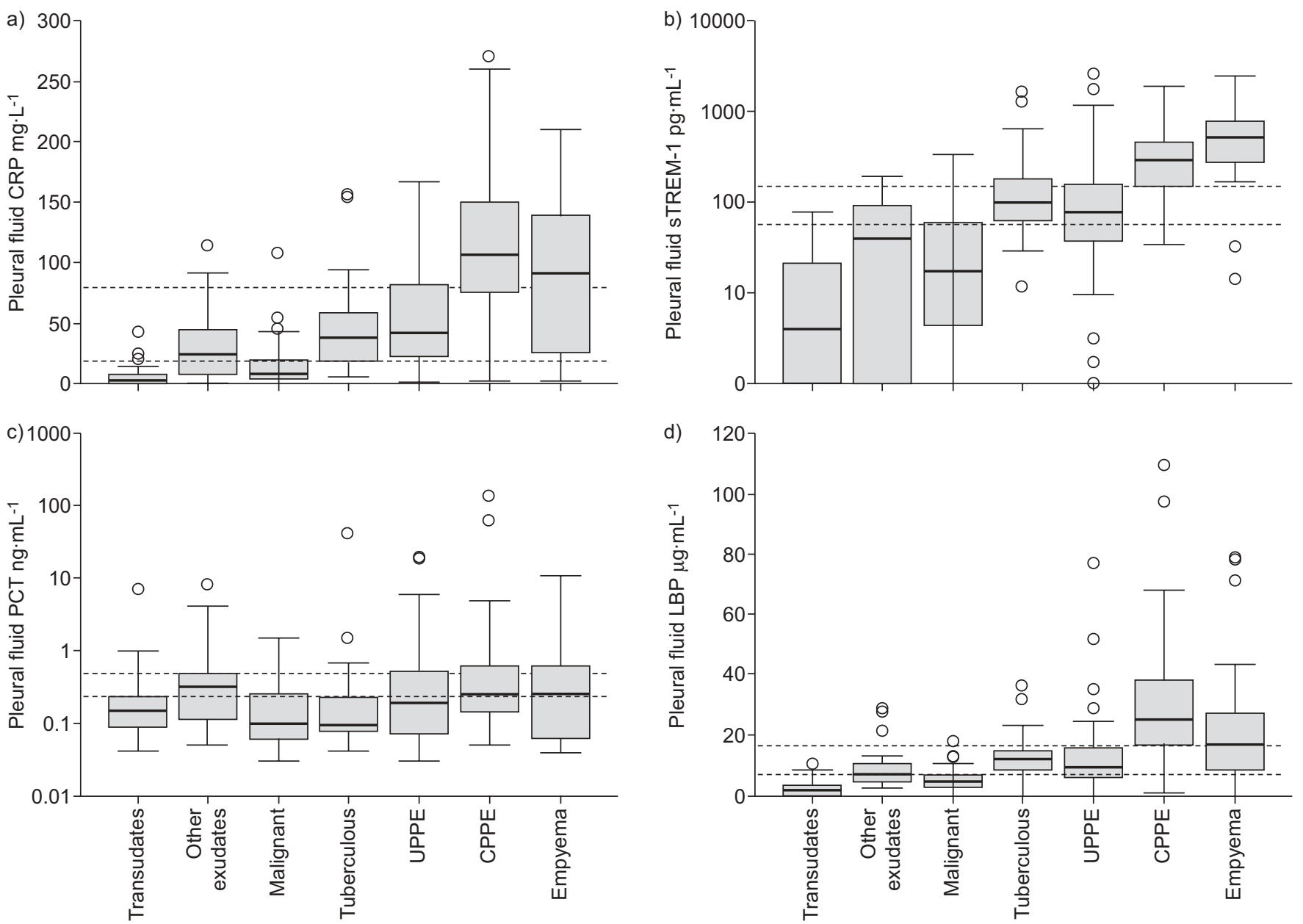

FIGURE 1. Box plots of pleural fluid concentrations of a) C-reactive protien (CRP), b) soluble triggering receptor expressed on myeloid cells (sTREM-1), c) procalcitonin (PCT) and d) lipopolysaccharide binding protein (LBP) in different aetiologies of pleural effusion. Pleural STREM-1 and PCT levels are plotted on a log scale. Horizontal dashed lines represent cut-off values with discriminatory properties. UPPE: uncomplicated parapneumonic effusion; CPPE: complicated parapneumonic effusion.

continuous variables, and Chi-squared tests with post hoc analysis of adjusted residuals for categorical variables. Receiver-operating characteristic (ROC) curves were constructed to illustrate the predictive value of various cut-off points of CRP, sTREM-1, PCT and LBP. The point with the largest sum of sensitivity and specificity was chosen as a threshold. We compared the performances of the four pleural fluid inflammatory biomarkers for discriminating infectious (bacterial and mycobacterial) versus noninfectious effusions, tuberculosis versus malignancy, PPE versus non-PPE, and CPPE versus UPPE. Measures of test efficacy included sensitivity, specificity and likelihood ratios. To adjust for confounders, a backward conditional stepwise logistic regression model estimated the simultaneous impact of each biomarker, along with other biochemical fluid findings, in the prediction of nonpurulent CPPE. The level of significance was set at $p<0.05$ using twotailed tests. Data were analysed using a statistical software package (SPSS version 13.0; SPSS Inc, Chicago, IL, USA).

\section{RESULTS}

Pleural fluid levels of the biomarkers

The demographic data and the median pleural fluid levels of CRP, sTREM-1, PCT and LBP in each of the seven groups are shown in table 1 . The levels of CRP, sTREM-1 and LBP were significantly higher in the pleural fluid from patients with CPPE and lower in the transudate and malignant groups (fig. 1). The levels of PCT showed the same trend but with a large overlap of values.

\section{Discriminating infectious from noninfectious effusions}

The thresholds of the four biomarkers that best discriminated between infectious (PPE and tuberculosis) and noninfectious effusions were as follows: CRP $\geqslant 20 \mathrm{mg} \cdot \mathrm{L}^{-1}$, sTREM- 1 $\geqslant 80 \mathrm{pg} \cdot \mathrm{mL}^{-1}$, PCT $\geqslant 0.25 \mathrm{ng} \cdot \mathrm{mL}^{-1}$ and $\mathrm{LPB} \geqslant 7 \mu \mathrm{g} \cdot \mathrm{mL}^{-1}$ (table 2). While PCT had no discriminative properties (area under curve (AUC) 0.57), sTREM-1 (AUC 0.86), CRP and LBP (both AUCs 0.87) showed good abilities to separate infectious from noninfectious states. However, the overlapping 95\% confidence interval values for these three biomarkers indicated that no single one could be identified as being superior to the others.

\section{Discriminating tuberculous from malignant effusions}

The AUCs for each pleural fluid biomarker (calculated with the cut-off points described above) in differentiating between tuberculous and malignant effusions are displayed in table 3. 
TABLE 2 Measures of diagnostic accuracy for pleural fluid tests that identify an infectious effusion\#

\begin{tabular}{|c|c|c|c|c|c|}
\hline & Sensitivity & Specificity & LR+ & LR- & AUC \\
\hline $\mathrm{CRP} \geqslant 20 \mathrm{mg} \cdot \mathrm{L}^{-1}$ & $83(78-88)$ & $76(67-85)$ & $3.5(2.4-4.9)$ & $0.22(0.16-0.31)$ & $0.87(0.84-0.91)$ \\
\hline PCT $\geqslant 0.25 \mathrm{ng} \cdot \mathrm{mL}^{-1}$ & $45(38-53)$ & $72(61-82)$ & $1.6(1.1-2.3)$ & $0.77(0.63-0.92)$ & $0.57(0.50-0.64)$ \\
\hline LBP $\geqslant 7 \mu \mathrm{g} \cdot \mathrm{mL}^{-1}$ & $83(77-88)$ & $78(69-87)$ & $3.8(2.6-5.6)$ & $0.22(0.16-0.30)$ & $0.87(0.82-0.91)$ \\
\hline
\end{tabular}

Data are presented as \% $(95 \% \mathrm{Cl})$. LR+: likelihood ratio positive; LR-: likelihood ratio negative; AUC: area under curve; CRP: C-reactive protein; sTREM-1: soluble triggering receptor expressed on myeloid cells; PCT: procalcitonin; LPB: lipopolysaccharide-binding protein. \#: infectious pleural effusion refers to parapneumonic and tuberculous aetiologies.

In contrast to PCT (AUC 0.52), which was not useful, CRP, sTREM-1 and LPB had better AUCs that were similar in value (all AUCs >0.80).

\section{Discriminating PPE from other aetiologies}

In discriminating PPE from the other aetiologies, the respective AUCs generated from the use of CRP, sTREM-1, PCT and LBP were $0.83,0.79,0.63$ and 0.80 (table 4 ). Therefore, PCT lacked efficacy as a marker of PPE.

\section{Discriminating UPPE from nonpurulent CPPE}

Table 5 shows the diagnostic accuracies of the four biomarkers in the differentiation of UPPE and nonpurulent CPPE at the optimum cut-off points. They were compared with the performances of $\mathrm{pH}$, glucose and $\mathrm{LDH}$ at the cut-off values recommended in the literature. All parameters, with the exception of PCT (AUC 0.59), performed well in discriminating between groups (AUCs $\sim 0.80$ ). Nevertheless, the overlap of the $95 \%$ confidence incidence values for the AUC and likelihood ratios did not allow for a selection of a superior test that clearly had the highest discriminative properties for nonpurulent CPPE.

When all the variables listed in table 5 were entered into a multivariate stepwise logistic regression model, the parameters that best discriminated nonpurulent CPPE from UPPE were as follows: glucose (adjusted likelihood ratio (LR)+ 5.2, 95\% CI 2.18; adjusted LR- 0.10, 95\% CI 0.02-0.44), LBP (adjusted LR+ 4.6, 95\% CI 2.1-7.1; adjusted LR- 0.14, 95\% CI 0.04-0.44) and CRP (adjusted LR+ 3.4, 95\% CI 1.4-6.1; adjusted LR- 0.22, 95\% CI 0.07-0.69). This model had an AUC of 0.88 (95\% CI 0.82-0.94).

\section{DISCUSSION}

A number of biochemical markers of bacterial infection have been identified, though the validity of their measurement in pleural fluid remains unclear. Our findings in a large series of patients provide data on the value and accuracy of some of these biomarkers (i.e. CRP, sTREM and LBP) measured in the pleural fluid to diagnose infectious effusions, particularly from CPPE.

\section{CRP}

CRP, an acute-phase reactant released from the liver, is a common diagnostic test within hospital laboratories for the screening or monitoring of infections and noninfectious inflammatory diseases. CRP levels have been studied in pleural fluid and have been found to be higher in PPEs than in other types of exudative or transudative effusions [5-7]. We showed that a pleural fluid CRP level $>80 \mathrm{mg} \cdot \mathrm{L}^{-1}$ argues for the presence of a PPE $(\mathrm{LR}+7.4)$, whereas CRP levels $<20 \mathrm{mg} \cdot \mathrm{L}^{-1}$ are a strong indicator against an infectious pleural effusion, whether of bacterial or mycobacterial nature (LR0.22). Indeed, a number of studies provide support for the use of CRP as a diagnostic aid in tuberculous pleuritis; low pleural CRP levels $\left(<30 \mathrm{mg} \cdot \mathrm{L}^{-1}\right)$ make this diagnosis unlikely while being more indicative of a malignancy in patients with exudates [8-10]. In addition, our findings complement the scarce previous literature on the application of CRP for identifying CPPE [11, 12]. This biomarker emerged as an independent predictor of nonpurulent CPPE in the multivariate analysis, although its adjusted $\mathrm{LR}+$ was lower than those for glucose and LBP.

TABLE 3 Diagnostic accuracy of individual pleural fluid tests for identifying tuberculous as compared with malignant effusions

\begin{tabular}{lccccc} 
& Sensitivity & Specificity & LR+ & LR- & AUC \\
\hline $\mathbf{C R P} \geqslant \mathbf{2 0} \mathbf{~ m g} \cdot \mathbf{L}^{-1}$ & $74(61-87)$ & $77(63-92)$ & $3.3(1.8-6)$ & $0.34(0.20-0.55)$ & $0.81(0.72-0.90)$ \\
$\mathbf{S T R E M}-\mathbf{1} \geqslant \mathbf{8 0} \mathbf{~ p g} \cdot \mathbf{m L}^{-1}$ & $62(47-76)$ & $85(73-97)$ & $4.1(1.9-8.9)$ & $0.45(0.31-0.65)$ & $0.83(0.74-0.92)$ \\
$\mathbf{P C T} \geqslant \mathbf{0 . 2 5} \mathbf{~ n g} \cdot \mathbf{m L}^{-1}$ & $25(12-39)$ & $76(60-91)$ & $1(0.5-2.2)$ & $0.98(0.77-1.26)$ & $0.52(0.39-0.65)$ \\
LBP $\geqslant \mathbf{7} \mathbf{\mu} \cdot \mathbf{m L}^{-1}$ & $79(67-92)$ & $77(63-92)$ & $3.5(1.9-6.4)$ & $0.27(0.15-0.48)$ & $0.83(0.74-0.92)$ \\
\hline
\end{tabular}

Data are presented as \% $(95 \% \mathrm{CI})$. LR+: likelihood ratio positive; LR-: likelihood ratio negative; AUC: area under curve; CRP: C-reactive protein; sTREM-1: soluble triggering receptor expressed on myeloid cells; PCT: procalcitonin; LPB: lipopolysaccharide-binding protein. 
TABLE 4 Diagnostic accuracy of individual pleural fluid tests for identifying parapneumonic effusions

\begin{tabular}{|c|c|c|c|c|c|}
\hline $\mathrm{CRP} \geqslant 80 \mathrm{mg} \cdot \mathrm{L}^{-1}$ & $49(41-57)$ & 93 (89-98) & $7.4(4-13.8)$ & $0.54(0.46-0.64)$ & $0.83(0.78-0.87)$ \\
\hline $\mathrm{PCT} \geqslant 0.25 \mathrm{ng} \cdot \mathrm{mL}^{-1}$ & $52(43-60)$ & $73(64-81)$ & $1.9(1.4-2.6)$ & $0.66(0.54-0.81)$ & $0.63(0.56-0.70)$ \\
\hline LBP $\geqslant 17 \mu \mathrm{g} \cdot \mathrm{mL}^{-1}$ & $49(40-57)$ & 90 (84-95) & $4.7(2.8-7.8)$ & $0.57(0.49-0.68)$ & $0.80(0.75-0.85)$ \\
\hline
\end{tabular}

Data are presented as \% $(95 \% \mathrm{Cl})$. LR+: likelihood ratio positive; LR-: likelihood ratio negative; AUC: area under curve; CRP: C-reactive protein; sTREM-1: soluble triggering receptor expressed on myeloid cells; PCT: procalcitonin; LPB: lipopolysaccharide-binding protein.

\section{STREM-1}

TREM-1, a receptor of the immunoglobulin superfamily, amplifies the inflammatory response through its over-expression and subsequent activation of neutrophils and monocytes/ macrophages in response to microbial products. TREM-1 is also shed by the membrane of activated phagocytes and can be found in a soluble form in body fluids.

Few studies have investigated the clinical significance of sTREM-1 in pleural effusions [13-18]. All included small sample sizes (45-109 patients), measured sTREM-1 by ELISA or other techniques and found that patients with PPE or empyema exhibited the highest pleural fluid concentrations of this biomarker. However, there were discrepancies regarding its discriminative properties as well as its optimal cut-off point. For example, in one study, an sTREM-1 cut-off value of $768.1 \mathrm{pg} \cdot \mathrm{mL}^{-1}$ had a sensitivity of $86 \%$, specificity of $93 \%$ and AUC of 0.93 in differentiating 23 bacterial effusions (including 17 empyemas) from 88 effusions with other aetiologies [17]. In contrast, our findings yielded an AUC of 0.79 for identifying PPE at the best cut-off of $80 \mathrm{pg} \cdot \mathrm{mL}^{-1}$. The over-representation of empyemas in the first series (74\% versus $19 \%$ of PPE) may help explain these discrepancies, while different sTREM-1 concentrations and cut-off points probably reflect the lack of standardisation of the ELISA technique. In a second study, a cut-off value of $114 \mathrm{pg} \cdot \mathrm{mL}^{-1}$ for pleural sTREM-1 achieved a sensitivity of $94 \%$ and a specificity of $93 \%$ (AUC 0.966 ) in differentiating 17 empyemas from 72 pleural effusions of other aetiologies [18]. We think there is no point in trying to identify empyema by measuring sTREM-1, because this diagnosis is easily achieved by simple inspection. Finally, in another study, pleural sTREM-1 at a cut-off value of $374 \mathrm{pg} \cdot \mathrm{mL}^{-1}$ yielded a sensitivity of $93.8 \%$, a specificity of $90.9 \%$ and an AUC of 0.93 in discriminating bacterial pleural infection $(n=22)$ from tuberculous pleuritis $(n=16)$ [14]. Based on our results (sensitivity $80 \%$, specificity $56 \%$ at a cut-off of $180 \mathrm{pg} \cdot \mathrm{mL}^{-1}$, AUC 0.64; data not shown), we do not support the use of sTREM-1 measurements for this particular indication. In addition, the ability to differentiate between PPEs and tuberculous effusions does not normally pose a problem in clinical practice.

Taken together, our data indicate that pleural fluid levels of sTREM-1 may be accurate enough to help differentiate between infectious and noninfectious effusions (AUC 0.86). Notably, two studies demonstrated that the levels of sTREM-1 in pleural fluid greatly exceeded those in serum [16, 17], suggesting that recruited inflammatory cells in the pleural space produce the sTREM-1 locally.

\section{Procalcitonin}

PCT, an acute-phase hormokine, has been reported to be more accurate for differentiating between bacterial infections and noninfectious causes of inflammation than other biomarkers [19]. Serum levels of PCT increase in severe bacterial infections. A PCT serum concentration of $0.5 \mathrm{ng} \cdot \mathrm{mL}^{-1}$ has been recommended in clinical practice guidelines to rule out bacterial infection [1] There is little useful information on PCT levels in pleural fluid.

TABLE 5 Operating characteristics of pleural fluid tests for distinguishing nonpurulent uncomplicated and complicated parapneumonic effusions

\begin{tabular}{|c|c|c|c|c|c|}
\hline & Sensitivity & Specificity & LR+ & LR- & AUC \\
\hline $\mathrm{pH} \leqslant 7.20$ & $57(44-70)$ & $93(85-100)$ & $8.1(3.1-21.5)$ & $0.46(0.34-0.62)$ & $0.83(0.75-0.91)$ \\
\hline Glucose $\leqslant 60 \mathrm{mg} \cdot \mathrm{dL}^{-1}$ & $59(46-71)$ & 90 (82-98) & $5.9(2.7-12.9)$ & $0.46(0.34-0.62)$ & $0.80(0.72-0.88)$ \\
\hline $\mathrm{LDH}^{\#} \geqslant 1000 \mathrm{U} \cdot \mathrm{L}^{-1}$ & $75(63-86)$ & $82(71-92)$ & $4.1(2.3-7.1)$ & $0.31(0.20-0.48)$ & $0.82(0.75-0.90)$ \\
\hline $\mathrm{CRP} \geqslant 80 \mathrm{mg} \cdot \mathrm{L}^{-1}$ & $68(56-79)$ & $75(63-87)$ & $2.7(1.7-4.3)$ & $0.43(0.30-0.63)$ & $0.81(0.63-0.88)$ \\
\hline sTREM-1 $\geqslant 180 \mathrm{pg} \cdot \mathrm{mL}^{-1}$ & $72(61-83)$ & $82(71-92)$ & $3.9(2.3-6.8)$ & $0.34(0.23-0.51)$ & $0.79(0.70-0.86)$ \\
\hline PCT $\geqslant 0.50 \mathrm{ng} \cdot \mathrm{mL}^{-1}$ & $33(21-45)$ & $72(59-86)$ & $1.2(0.7-2.1)$ & $0.93(0.73-1.17)$ & $0.59(0.49-0.70)$ \\
\hline LBP $\geqslant 17 \mu \mathrm{g} \cdot \mathrm{mL}^{-1}$ & 76 (64-87) & 81 (70-92) & $4(2.3-6.9)$ & $0.30(0.19-0.47)$ & $0.84(0.76-0.91)$ \\
\hline
\end{tabular}

Data are presented as \% $(95 \% \mathrm{Cl})$. LR+: likelihood ratio positive; LR-: likelihood ratio negative; AUC: area under curve; LDH: lactate dehydrogenase; CRP: C-reactive protein; sTREM-1: soluble triggering receptor expressed on myeloid cells; PCT: procalcitonin; LPB: lipopolysaccharide-binding protein. \#: in our laboratory, the normal upper limit for serum $\mathrm{LDH}$ is $470 \mathrm{U} \cdot \mathrm{L}^{-1}$. 
One study found that the median pleural fluid PCT levels were significantly higher in 26 patients with bacterial infection $\left(0.67 \mathrm{ng} \cdot \mathrm{mL}^{-1}\right)$ than in 80 malignant effusions $\left(0.14 \mathrm{ng} \cdot \mathrm{mL}^{-1}\right), 33$ cardiac effusions $\left(0.06 \mathrm{ng} \cdot \mathrm{mL}^{-1}\right)$ and 17 viral effusions $\left(0.007 \mathrm{ng} \cdot \mathrm{mL}^{-1}\right)$ [20]. However, the authors did not fully describe the diagnostic criteria of the study population or calculate any discriminatory cut-off values for PCT. A recent investigation showed that pleural fluid PCT levels $>0.18 \mathrm{ng} \cdot \mathrm{mL}^{-1}$ discriminated 45 PPEs from 37 non-PPEs with a sensitivity of $67 \%$, a specificity of $77 \%$ and an AUC of 0.752 [21]. Interestingly, serum PCT had better diagnostic accuracy than pleural PCT and both correlated with the severity of pneumonia.

Our study failed to demonstrate any firm relationship between pleural fluid levels of PCT and the specific cause of the pleural effusion. These disappointing results could be explained by considering PCT as a potential biomarker of a state or a syndrome (e.g. severe sepsis) rather than an indicator of a disease. In fact, some studies have reported that, as a marker of bacterial infection, PCT was no better than CRP [22]. However, in itself, it was considered quite useful as a marker regarding the severity of infection.

\section{$L B P$}

LBP, a glycosylated 58-kDa protein produced predominantly in hepatocytes, has recently been described as a promising novel diagnostic marker of bacterial infection [22]. To the best of our knowledge, pleural fluid LBP concentrations have not been previously evaluated. We observed that pleural fluid LBP levels of $<7 \mu \mathrm{g} \cdot \mathrm{mL}^{-1}$ substantially reduced the probability of an infectious aetiology (LR- $=0.22$ ). This may have implications, for instance, in the differential diagnosis of tuberculous and malignant effusions. Moreover, a LBP $>17 \mu \mathrm{g} \cdot \mathrm{mL}^{-1}$ conferred an adjusted LR+ of 4.6 in identifying nonpurulent CPPE, a performance that was similar to the currently used tests, such as glucose or $\mathrm{pH}$.

\section{Selecting tests}

The choice of one among different diagnostic tests depends not only on its accuracy, but also on the financial costs and technical considerations. The cost of one measurement for CRP, sTREM-1, PCT and LBP is about $1,4,14$ and $6 €$, respectively. Conversely, CRP and LBP measurements are less labour-intensive than those for PCT and sTREM-1. Therefore, given the lack of utility of measuring PCT and the similarity in the accuracy of the other three tests, cost-effectiveness and technical reasons would argue for the diagnostic use of CRP, if a selection needs to be done.

\section{Study limitations}

This study should be interpreted in the context of certain limitations. First, the use of physician judgment as the gold standard for indicating the need for pleural drainage in CPPE may have resulted in patients' misclassifications, thus compromising the diagnostic accuracy of the pleural fluid tests. However, satisfactory correction for the bias introduced by an imperfect gold standard does not exist. Secondly, except for the identification of nonpurulent CPPE, we have not compared the advantages of the new tests over alternative tests; that is, we have not answered the question of whether the new tests are better than the current diagnostic standard. Nevertheless, our primary goal was simply to determine whether the new biomarkers accurately and reliably identified cases of obvious infectious pathology in a well-defined population. Whether these biomarkers offer additional information beyond that which is normally available is a matter of speculation. Finally, our findings, which are based on a retrospective analysis, deserve further prospective evaluation in an independent validation cohort.

\section{Conclusions}

In summary, CRP, sTREM-1 and LBP measurements may be useful as an additional tool in the assessment of pleural effusions to support the differential diagnosis between infectious and noninfectious aetiologies. However, in the most challenging clinical setting, such as the identification of a nonpurulent PPE needing a tube thoracostomy, the accuracy of the three biomarkers is comparable with that of the classical pleural fluid biochemistries, such as $\mathrm{pH}$, glucose or LDH. For this reason, we predict that even if these new tests are adopted, they will not have a major impact on clinical practice.

\section{SUPPORT STATEMENT}

This study was supported by a grant from the Instituto de Salud Carlos III, Madrid, Spain (FIS 06/0725). S. Bielsa is a Research Fellow supported by a grant from the Fondo de Investigación Sanitaria, Madrid (FIS CM07/00020).

\section{STATEMENT OF INTEREST}

None declared.

\section{REFERENCES}

1 Christ-Crain M, Müller B. Biomarkers in respiratory tract infections: diagnostic guides to antibiotic prescription, prognostic markers and mediators. Eur Respir J 2007; 30: 556-573.

2 Rahman NM, Davies RJO. Effusions from infections: parapneumonic effusion and empyema. In: Light RW, Gary Lee YC, eds. Textbook of Pleural Diseases. Hodder Arnold, London, 2008; pp. 341-366.

3 Herzum I, Renz H. Inflammatory markers in SIRS, sepsis and septic shock. Curr Med Chem 2008; 15: 581-587.

4 Sharif O, Knapp S. From expression to signaling: roles of TREM-1 and TREM-2 in innate immunity and bacterial infection. Immunobiology 2008; 213: 701-713.

5 Yilmaz Turay U, Yildirim Z, Türköz Y, et al. Use of pleural fluid Creactive protein in diagnosis of pleural effusions. Respir Med 2000; 94: 432-435.

6 García-Pachon E, Llorca I. Diagnostic value of C-reactive protein in exudative pleural effusions. Eur J Intern Med 2002; 13: 246-249.

7 Kiropoulos TS, Kostikas K, Oikonomidi S, et al. Acute phase markers for the differentiation of infectious and malignant pleural effusions. Respir Med 2007; 101: 910-918.

8 Chierakul N, Kanitsap A, Chaiprasert A, et al. A simple C-reactive protein measurement for the differentiation between tuberculous and malignant pleural effusion. Respirology 2004; 9: 66-69.

9 Garcia-Pachon E, Soler MJ, Padilla-Navas I, et al. C-reactive protein in lymphocytic pleural effusions: a diagnostic aid in tuberculous pleuritis. Respiration 2005; 72: 486-489.

10 Daniil ZD, Zintzaras E, Kiropoulos T, et al. Discrimination of exudative pleural effusions based on multiple biological parameters. Eur Respir J 2007; 30: 957-964.

11 Chen SC, Chen W, Hsu WH, et al. Role of pleural fluid C-reactive protein concentration in discriminating uncomplicated parapneumonic pleural effusions from complicated parapneumonic effusion and empyema. Lung 2006; 184: 141-145. 
12 Porcel JM, Galindo C, Esquerda A, et al. Pleural fluid interleukin-8 and C-reactive protein for discriminating complicated nonpurulent from uncomplicated parapneumonic effusions. Respirology 2008; 13: 58-62.

13 Liu CL, Hsieh WY, Wu CL, et al. Triggering receptor expressed on myeloid cells-1 in pleural effusions: a marker of inflammatory disease. Respir Med 2007; 101: 903-909.

14 Chan MC, Chang KM, Chao WC, et al. Evaluation of a new inflammatory molecule (triggering receptor expressed on myeloid cells-1) in the diagnosis of pleural effusion. Respirology 2007; 12: 333-338.

15 Kim JH, Park EY, Kim WH, et al. Soluble triggering receptor expressed on myeloid cells-1: role in the diagnosis of pleural effusions. Tuberc Respir Dis 2007; 62: 290-298.

16 Sim YS, Lee JH, Chun EM, et al. Diagnostic utility of pleural fluid soluble triggering receptor expressed on myeloid cells 1 in patients with exudative pleural effusion. Tuberc Respir Dis 2007; 62: 499-505.
17 Huang LY, Shi HZ, Liang QL, et al. Expression of soluble triggering receptor expression on myeloid cells-1 in pleural effusion. Chin Med J (Engl) 2008; 121: 1656-1661.

18 Bishara J, Goldberg E, Ashkenazi S, et al. Soluble triggering receptor expressed on myeloid cells-1 for diagnosing empyema. Ann Thorac Surg 2009; 87: 251-254.

19 Becker KL, Snider R, Nylen ES. Procalcitonin assay in systemic inflammation, infection, and sepsis: clinical utility and limitations. Crit Care Med 2008; 36: 941-952.

20 Topolcan O, Holubec L, Polivkova V, et al. Tumor markers in pleural effusions. Anticancer Res 2007; 27: 1921-1924.

21 Lin MC, Chen YC, Wu JT, et al. Diagnostic and prognostic values of pleural fluid procalcitonin in parapneumonic pleural effusions. Chest 2009; 136: 205-211.

22 Gaïni S, Koldkjaer OG, Pedersen C, et al. Procalcitonin, lipopolysaccharide-binding protein, interleukin-6 and C-reactive protein in community-acquired infections and sepsis: a prospective study. Crit Care 2006; 10: R53. 